\title{
Pero... ¿A qué nos Referimos Realmente con la Evaluación Formativa y Compartida?: Confusiones Habituales y Reflexiones Prácticas
}

\author{
But... What do we Really mean by Formative and Shared \\ Assessment?: Usual Confusions and Practical Reflections
}

\author{
David Hortigüela $* 1$ \\ Ángel Pérez-Pueyo ${ }^{2}$ \\ Gustavo González-Calvo ${ }^{3}$ \\ ${ }^{1}$ Universidad de Burgos, España \\ ${ }^{2}$ Universidad de León, España \\ ${ }^{3}$ Universidad de Valladolid, España
}

\begin{abstract}
Es fundamental reflexionar sobre los fines y posibilidades del uso de la evaluación formativa y compartida (EFyC) en los contextos educativos. Si bien la literatura acerca de sus efectos en diversidad de variables es abundante y prolija, no lo es tanto a la hora de aclarar determinadas confusiones pedagógicas en su puesta en práctica. Esto se hace necesario, más si cabe en la actualidad, donde su difusión ha sido muy elevada en los últimos años. Los objetivos del presente estudio son dos: a) presentar los errores más habituales a la hora de emplear la EFyC y b) establecer reflexiones y consideraciones prácticas para su buena utilización. Los ejes temáticos analizados son: 1) evaluación formativa/calificación continua; 2) el docente como único agente de feedback; 3) no delimitación de los criterios de antemano; 4) no permitir entregas previas; 5) no garantizar el salto a la calificación de manera pedagógica; 6) no asociar procedimientos de evaluación con sus respectivos instrumentos; 7) utilizar la evaluación con fines puramente académicos. Se pretende que este manuscrito sea de especial interés y utilidad para todos los docentes interesados en el ámbito de la evaluación, y como consecuencia en mejorar su práctica educativa. Es preciso recordar que la educación es mucho más que la evaluación, pero sin esta última, sus efectos son más limitantes.
\end{abstract}

Palabras clave: Evaluación formativa y compartida; Reflexión docente; Implicación del alumnado; Aprendizaje activo.

It is fundamental to reflect on the aims and possibilities of the use of formative and shared evaluation (FSA) in educational contexts. Although the literature about its effects on diversity of variables is abundant and tedious, it is not so much at the time of clarifying certain pedagogical errors in its implementation. This is necessary, even more so now, where its dissemination has been very high in recent years. The objectives of this study are two: a) to present the most common confusions when using FSA and b) to establish reflections and practical considerations for its proper use. The topics analyzed are: 1) formative assessment/continuous qualification; 2) the teacher as the only feedback agent; 3) no delimitation of the criteria in advance; 4) not allow previous deliveries; 5) not guarantee the jump to the qualification in a pedagogical way; 6) not to associate evaluation procedures with their respective instruments; 7) use the evaluation for purely academic purposes. It is intended that this manuscript may be of special interest and usefulness for all teachers interested in the field of evaluation, and as a consequence to improve their educational practice. It must be remembered that education is much more than assessment, but without it, its effects are more limiting.

Keywords: Formative and shared assessment; Teacher reflection; Involvement of students; Active learning.

*Contacto: dhortiguela@ubu.es

issn: 1989-0397

www.rinace.net/riee/

https://revistas.uam.es/riee
Recibido: $\quad 28$ de diciembre de 2018

$1^{\text {a }}$ Evaluación: 28 de febrero de 2019

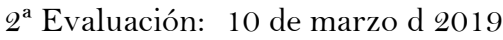

Aceptado: $\quad 16$ de marzo de 2019 


\section{Introducción}

A pesar de que podamos creer que es un tema ya superado, en multitud de ocasiones, cuando hablamos de evaluación, realmente se está hablando de calificación. Esta confusión, que parece no tener demasiada transcendencia, conlleva una serie de actuaciones en el aula no del todo pedagógicas. Como indican Chiappe, Pinto y Arias (2016), el término global de evaluación debería de asociarse al de aprendizaje, y para ello es necesario dotarlo de plena intencionalidad en el proceso de enseñanza. Todo lo calificable debería de ser evaluable pero no todo lo evaluable tiene por qué ser calificable. Esta frase refleja perfectamente las diferencias entre evaluación y calificación, entendida esta última como la delimitación de una nota numérica al finalizar un proceso y sobre la que no se ha constatado intencionadamente el aprendizaje adquirido (Fraile, López-Pastor, Castejón y Romero, 2013). Deberíamos de evaluar más y calificar menos, y ya que tenemos que calificar en cumplimiento con los aspectos legales, por lo menos hacerlo garantizando unas condiciones evaluativas previas coherentes (Hortigüela, Pérez-Pueyo y Abella, 2015a).

Es en esta dicotomía donde cobra especial relevancia la EFyC, definiéndose como el proceso de retroalimentación entre todos los agentes educativos, sin asociarse a una calificación, y con el fin de mejorar tanto el aprendizaje de los estudiantes como la propia práctica docente (Gutiérrez, Hortigüela, Peral y Pérez-Pueyo, 2018). Este enfoque evaluativo, que, si bien no es el predominante en el ámbito educativo, ha experimentado un avance notorio en los últimos años. Tanto en el ámbito nacional como en el internacional, son infinidad las experiencias, en todos los niveles educativos, las que han sido difundidas. En concreto, en nuestro país, y a través de la Red de Evaluación Formativa y Compartida, se celebran jornadas anuales y congresos internacionales bianuales en los que participan gran cantidad de docentes en diversidad de ámbitos ${ }^{1}$. A su vez, y desde los centros de formación del profesorado, se han impartido en los últimos años diversidad de cursos vinculados a la evaluación y metodologías activas o la evaluación de las competencias. Esto hace, sin duda, que hablar de evaluación formativa y la implicación del estudiante en su evaluación ya no parezca tan extraño.

Sin embargo, y aunque esto haya supuesto muchos avances respecto a los tratamientos de evaluación más tradicionales, todavía queda mucho por hacer. Es todavía habitual comprobar cómo la evaluación, más bien entendida como calificación, es utilizada por los docentes como herramienta de poder. Además, esto se hace desde una lógica subconsciente imbuida en muchos casos en el propio currículo oculto, partiendo de premisas tan ilógicas como asumidas como la de que el docente es el único que "sabe" (Shepard, Penuel y Pellegrino, 2018). Los docentes reconocen en muchos casos que se sienten mucho más seguros cuando ellos controlan la evaluación del estudiante, y en cierto modo pueden decidir exclusivamente la calificación que merecen. Es coincidente que los que aplican esta lógica también se alejan de la comprensión de la evaluación en sentido más global, no otorgando intencionalidad a la del profesorado y a la del proceso de enseñanza aprendizaje (Serrano, 2010).

\footnotetext{
${ }^{1}$ https://revistas.uv.cl/index.php/IEYA/issue/view/88/showToc.
} 
Aquí se abre un campo con infinidad de posibilidades, teniendo presente que la evaluación ha de ser compartida y democrática. ¿Cómo queremos que alguien se implique al máximo en algo cuando no puede controlar su proceso de aprendizaje? ¿Cómo queremos que el estudiante sea plenamente consciente de lo que aprende si no le garantizamos el mayor número y mejor calidad de feedbacks posibles? Si de verdad queremos que factores como la autorregulación del trabajo, la autonomía o la metacognición entren en juego, es fundamental que la evaluación sea transparente, clara, y que permita la decisión de todos los agentes que la sustentan, en este caso, dando voz a los estudiantes (Hortigüela, PérezPueyo y Abella, 2015b). A veces menos, es más, por lo que en muchas ocasiones puede tener más sentido pedagógico demandar un menor número de actividades evaluativas, pero con más transcendencia, y sobre todo, más transversales. Si partimos de la base de que las relaciones sociales a través del diálogo, la creatividad, la reflexión crítica y la colaboración han de estar presentes en nuestras aulas por la transcendencia que tienen en la sociedad actual, hemos de usar la evaluación como herramienta de autoconocimiento y mejora de todos los aspectos educativos (Calkins et al. 2018)

Es por ello que el concepto puro de evaluar debiera de estar asociado al de aprender. Sin una evaluación abierta, clara, participativa y democrática el aprendizaje nunca alcanzará su máximo nivel. Además, hay que destacar que la evaluación, dentro de un sistema educativo, no repercute únicamente en el estudiante, sino que la evaluación de la práctica docente y la del proceso de enseñanza han de estar presentes. No podemos olvidar que, en una sociedad tan cambiante como la actual en relación a la manera de recibir y acceder la información, la forma de interaccionar con los demás e incluso la manera de concebir los propios fines de la educación formal, los profesionales de la educación hemos de adentrarnos en profundos procesos de reflexión sobre cuál es nuestro rol en el aula. ¿No nos encontramos ante una pirotécnica metodológica constante? ¿Es que si no aplico todas las metodologías no soy tan buen docente o no estoy en la onda de "innovación"? Ante esto, es preciso recordar que la verdadera innovación es la que impacta en nuestras aulas y permite a nuestros estudiantes ser competentes. Eso sí, pensemos en la metodología que pensemos, la evaluación siempre está presente. Depende de cómo la usemos y los agentes que queramos que participen en la misma, así será la verdadera proyección de aquello que enseñemos.

Por lo tanto, y a partir de lo anteriormente comentado, los objetivos del presente estudio son dos: a) presentar los errores más habituales a la hora de emplear la $\mathrm{EFyC} \mathrm{y} \mathrm{b)}$ establecer reflexiones y consideraciones prácticas para su buena utilización.

\section{2. ¿Por qué decantarse por aplicar estos procesos evaluativos en el aula?}

Abordaremos este apartado destacando las cinco razones fundamentales por las que, en función de la literatura, es necesario aplicar procesos de evaluación formativa:

- Permite ser más consciente de lo que se aprende: no hay aprendizaje si no hay consciencia previa sobre aquello se está trabajando. Para ello, es fundamental delimitar los criterios de evaluación de antemano, e incluso dejar al estudiante que participe en la elaboración de los mismos (Wang, 2017). Del mismo modo, es fundamental tener clara la diferencia entre procedimiento e instrumento de 
evaluación, siendo estos últimos las herramientas que permiten plasmar los resultados de aprendizaje, no teniendo por qué asociarse a un número ni por supuesto establecerse en un momento final. Esta idea es imprescindible asociarla a la del concepto de reto educativo, y si es en grupo, mejor. Aquí, el aprendizaje cooperativo juega un papel relevante, entendiendo que, si asumimos tanto la responsabilidad individual como grupal dentro de un proyecto, y si por lo tanto sacamos lo mejor de cada uno, las posibilidades de que se genere motivación intrínseca aumentan considerablemente (Keely, 2016).

- Capacidad para autorregular el aprendizaje: la evaluación formativa y la autorregulación van estrechamente dadas de la mano. Las metodologías basadas en la capacidad del estudiante para controlar y decidir sobre sus tiempos de actuación y de trabajo han permitido un mayor impacto educativo (Delgado, Hortigüela, Ausín y Abella, 2018). Esto requiere cambiar el enfoque de actuación en el día a día y la exclusiva dependencia del libro de texto. ¿Por qué un niño tiene que hacer una tarea sistemáticamente para el día siguiente? ¿No puede decidir él sobre el cuándo? ¿Es que nosotros como adultos no organizamos nuestras tareas diarias? La capacidad organizativa del estudiante ya ha demostrado unos mejores resultados de aprendizaje, y para ello los proyectos de trabajo y la evaluación asociada al proceso se tornan claves (Meusen-Beekman, Joosten-ten Brinke y Boshuizen, 2015). Pero no nos olvidemos de que tanto el establecimiento de compromisos por parte del alumno y profesor como la asunción de responsabilidades hacia el trabajo es algo indisociable al propio éxito escolar, algo que favorece también la atención individualizada y su posterior intervención hacia las necesidades educativas especiales.

- Diferentes capacidades y competencias en el aprendizaje: una de nuestras principales responsabilidades como docentes se encuentra en conseguir transformar capacidades en competencias. Estas competencias demuestran la capacidad de saber solventar problemas, incluso de predecir algunos y actuar en consecuencia. En este sentido, y al entender la evaluación desde un prisma abierto, global y plenamente transversal, se les otorga a los estudiantes los medios para que puedan decidir el grado de implicación individual y de grupo hacia las tareas demandadas, reconociendo tanto sus potencialidades como sus limitaciones (Joughin, Dawson y Boud, 2017). No depende tanto de cuántos contenidos instrumentales se han adquirido en un determinado curso, sino de lo que se es capaz de hacer con los mismos. Para ello, técnicas como las coevaluaciones intra o intergrupales les harán ser conscientes del grado de adquisición de lo aprendido en función de la percepción de los demás, y lo que es más importante, de la manera en la que pueden aplicarlo fuera del aula (López-Pastor y Sicilia-Camacho, 2017).

- Diversidad de canales de feedback: la aplicación de la EFyC amplía el espectro de visión sobre el que se analiza el aula. No solamente es el docente el que da la información, corrige o aconseja, sino que cada integrante tiene la oportunidad de mejorar aquello sobre lo que se esté trabajando. De este modo, y bajo la esencia de grupo, la evaluación, unida al uso de metodologías activas y participativas, permite a cada estudiante dar valoraciones, constructivas y sistemáticas, tanto a su trabajo como al de los demás (Wanner y Palmer, 2018). Esta transparencia 
evaluativa favorece el empleo de diferentes canales de retroalimentación: orales, escritos, en vídeo, individuales, grupales..., y lo más interesante, aplicados por todos y todas de manera motivante. Los enfoques más tradiciones de evaluación dejan de lado el avance compartido, el diálogo consensuado y la verdadera interacción, tanto entre estudiantes como entre docente y discentes, algo que no tiene demasiado sentido en la concepción educativa actual.

- Mejora de la práctica docente: es de especial relevancia remarcar que la EFyC no se enmarca únicamente en el alumnado. Además, esto es debido a una principal y categórica razón: porque la evaluación nunca debiera de hacerlo. Si entendemos la evaluación como la manera más efectiva para mejorar los procesos educativos, debiéramos de valorar de un modo sistemático cuál es el verdadero impacto que nuestra docencia tiene en los estudiantes, y, por ende, cómo nosotros podemos mejorar nuestra docencia (Wei, 2014). Procedimientos como la observación sistemática y los intercambios orales con los alumnos, e instrumentos como rúbricas, escalas de valoración u hojas de registro, pueden favorecer el conocimiento y el intercambio de información sobre variables como la motivación del estudiante, el clima de grupo, el control sobre el aula, la percepción de lo aprendido y/o las dificultades para desarrollar ciertos contenidos. Si para aprender se necesita una buena fuente de enseñanza, impartida bajo un sistema educativo de calidad, es necesario tener abiertos diversos canales de retroalimentación para seguir avanzado de un modo colaborativo (Hortigüela, Palacios y López-Pastor, 2018).

\section{Algunos errores más habituales}

Atendiendo de nuevo a la literatura existente sobre la temática, y una vez destacadas las ventajas de aplicar sistemas de EFyC en el aula, se presentan a continuación los siete errores más habituales a la hora de aplicar estos procesos. Es pertinente tenerlos en cuenta, ya que, si los cometemos, además de desvirtuar la evaluación como elemento de aprendizaje, estaremos quebrantando la esencia educativa que tantos beneficios ya ha demostrado científicamente.

1. Evaluación formativa/calificación continua: para empezar, evaluación formativa y continúa no es lo mismo, y es algo que suele confundirse habitualmente. El problema es que se están mezclando dos criterios diferentes. Si atendemos al momento en el que se desarrolla la evaluación hablamos de inicial, continua y final. Si nos referimos al para qué de la evaluación nos encontramos con la diagnóstica, formativa y sumativa. Es atendiendo al para qué donde la evaluación cobra toda su dimensión, ya que nos permite intervenir en relación a la misma. Definida con anterioridad la evaluación formativa, otra confusión habitual suele ser asociarla a la evaluación continua, o lo que es peor, a la calificación continua. Una evaluación continua es por ejemplo el típico examen parcial, que, si se aprueba resta materia para el siguiente, pero no tiene por qué atender a un análisis de los errores para la mejora. Sin embargo, la EFyC atiende exclusivamente a esa retroalimentación para, sin asociarse a una calificación, buscar dos fines fundamentales: a) ser consciente de los puntos fuertes y débiles de lo trabajado y b) establecer los 
mecanismos y las herramientas de actuación para alcanzar esa mejora (Purcell, $2014)$.

2. El docente como único agente de feedback: aunque muchos docentes parezcan tener claro el uso sistemático y los fines de la $\mathrm{EFyC}$, no la abordan bajo la participación democrática de los agentes educativos que la integran (Lorente-Catalán y Kirk, 2014). En este sentido, y aunque sí que utilizan la retroalimentación, desde un enfoque constructivo, para la mejora del conocimiento, siguen siendo ellos como profesores los que llevan el mayor peso (en muchos casos exclusivo) de dichos feedbacks. Sin embargo, esta pierde fuerza cuando no está integrada e interrelacionada con la de los estudiantes (Alcaraz, Navas y Solac, 2012). Así, a la combinación entre la autoevaluación individual y/o en grupo la coevaluación intra y/o intergrupal y la heteroevaluación del docente es denominada como evaluación triádica. Para poder desarrollarla, es necesario tener dos aspectos fundamentales en cuenta: a) que se lleve a cabo a través de procesos de $\mathrm{EFyC} \mathrm{y} \mathrm{b)} \mathrm{que} \mathrm{se} \mathrm{realice}$ en un mismo instrumento de evaluación (rúbrica, escala de valoración, lista de control...) atendiendo a un procedimiento de evaluación concreto.

3. No delimitación de los criterios de antemano: quizás este se convierta en uno de los errores que, si no es realizado con plena consciencia y transparencia, tenga más efectos negativos en la docencia, y como consecuencia en la evaluación (Alonzo, 2018). Cualquier tipo de retroalimentación carece de sentido y efecto si no se estructura bajo un conocimiento previo de lo que se demanda. Esto parece obvio, pero la realidad no refleja lo mismo. Si bien la observación sistemática es uno de los principales procedimientos de evaluación para controlar y garantizar el proceso de enseñanza, una mala comprensión y uso del mismo puede tener consecuencias nefastas. ¿Se siguen poniendo las notas a ojímetro? ¿Conoce el estudiante los instrumentos con los que será evaluado? ¿Participa en los mismos a lo largo del proceso? Que el docente conozca la evolución del alumnado no está reñido con el uso de instrumentos conocidos y trabajados de antemano. Afirmaciones como "yo con verles ya se lo que aprenden" o "es que no se ha esforzado lo suficiente" (sin delimitar a lo que nos referimos con esfuerzo) no nos permiten avanzar hacia un sistema educativo abierto, democrático y transparente para todos. Si bien parece que en los últimos años el uso de instrumentos de evaluación por parte del docente es más extendido, no lo es así la intencionalidad en uso. Hemos pasado de una época en la que no se usaba ningún instrumento a otra en la que suelen utilizarse, pero sin tener siempre en cuenta al alumnado para su interiorización y trabajo. ¿De qué sirve que el docente utilice una rúbrica para evaluar y calificar un trabajo si no se permite durante el proceso que esta sea utilizada mediante procesos de evaluación formativa? A mayor utilización, mayores posibilidades de que exista consciencia sobre lo que se va trabajando, y como consecuencia, mayores niveles de aprendizaje adquirido (Wilkie, 2016).

4. No permitir entregas previas: la aplicación de EFyC tiene que garantizar la emisión de feedbacks periódicos y efectivos. Entendemos por efectividad aquellos que son asumidos por el sujeto que ejecuta las tareas. Bajo este prisma de visión es necesario buscar tareas de calidad asociadas al desarrollo de competencias. Cano (2008) indica que, en diversidad de ocasiones, y debido al cumplimiento cultural y subconsciente del currículo, los docentes demandan un exceso de tareas de escasa 
relevancia en el aprendizaje competencial del estudiante (Ashgar, 2010). Ante esto, una buena tarea debiera de cumplir por lo menos estos tres requisitos: a) que suponga un reto para el estudiante, favoreciendo así la motivación intrínseca para su resolución; b) que favorezca la colaboración y el diálogo entre iguales y c) que conexione con las demandas sociales, siendo transferible a diversidad de ámbitos. Todo esto ha de ser articulado mediante la revisión previa del trabajo realizado, con el fin de garantizar que todo se esté desarrollando adecuadamente, y si no es así, poner herramientas para la reconducción. Hay que tener presente que estas entregas previas a la calificación final, recomendable al menos hacer dos, pueden ser de todo tipo: escritas, orales, audiovisuales...lo que da más valor aún si cabe a la evaluación formativa.

5. No garantizar el salto a la calificación de manera pedagógica: en determinadas ocasiones, y aunque algunos docentes realizan procesos de evaluación formativa de manera periódica, existe cierta desconexión entre esta y el salto a la calificación. Es de suma importancia encontrar la linealidad en los procesos de evaluación (Grosas, Raju, Schuett, Chuck y Millar, 2016). Suele ser habitual utilizar procesos de calificación finales sin haber establecido las condiciones evaluativas acordes previas, lo que supone una incoherencia en el proceso de enseñanza y la generación de lagunas de aprendizaje a diversos niveles. En esta línea, puede incurrirse en algunos aspectos discordantes, como aplicar procesos de EFyC en determinados procedimientos de evaluación y luego pasar a la calificación sin tener presente los acuerdos tomados previamente (Hortigüela, Fernández-Río, Castejón y PérezPueyo, 2017). Para evitar esto, es imprescindible que los compromisos alcanzados entre estudiantes y docente en las retroalimentaciones establecidas tengan una repercusión a la hora de pasar a la calificación. Así, técnicas como el reparto de notas o la calificación dialogada han de estar sustentadas bajo principios y acuerdos coherentes en función de la responsabilidad adquirida por el estudiante.

6. No asociar procedimientos de evaluación con sus respectivos instrumentos: si ya dejamos clara la diferencia entre ambos, es importante establecer metodologías que permitan asociarlos. En primer lugar, es necesario tener presente que cada procedimiento de evaluación ha de tener asociado un instrumento, de este modo sabemos con qué herramientas daremos la retroalimentación y podremos dar el salto a la calificación final. Es el uso del instrumento lo que marca la diferencia en relación a la adquisición de procesos metacognitivos por parte del estudiante (Klenowski, Askew y Carnell, 2006). Estos instrumentos, pueden ser de diferentes tipos y usados a través de distintas vías, analógicas o digitales. Los más conocidos suelen ser las rúbricas, las escalas de valoración, las hojas de observación y las listas de control. Lo más relevante aquí es que integren aquellas variables dependientes (aspectos a valorar) que queremos medir y controlar, así como los indicadores de logro (rangos de nivel de cada aspecto a valorar). A modo de ejemplo, si como procedimiento utilizaremos el análisis de producciones de los alumnos, en concreto, la exposición oral de un trabajo realizado, como instrumento podremos emplear una escala de valoración. En esta escala podemos incluir variables como: el uso de las manos y el cuerpo, la entonación, la fluidez o la inclusión de los contenidos vistos en la asignatura. Otro aspecto a destacar en este sentido es la necesidad de aplicar variedad de procedimientos de evaluación a 
lo largo del curso, lo que aumenta la riqueza de registros y capacidades a emplear por los estudiantes (López-Pastor, 2008). Esto no tiene que convertirse en una saturación para el docente, ya que estaríamos incumpliendo uno de los principales principios de la $\mathrm{EFyC}$, como es la viabilidad.

7. Utilizar la evaluación con fines puramente académicos: Si la literatura ya ha demostrado que el componente social en el aula es uno de los factores que más incide en el aprendizaje, la evaluación no puede permanecer al margen. Sería un error entender y dirigir todo el potencial educativo de la $\mathrm{EFyC}$ hacia fines puramente académicos. Cuando en el aula se establece un buen clima de trabajo orientado hacia la tarea, enmarcado bajo la naturalidad de un diálogo construido a partir de críticas constructivas, el aprendizaje es más reflexivo y duradero. Este enfoque, basado en la necesidad y relevancia que tienen las relaciones sociales, favorece el conocimiento propio y el de los demás, tomando decisiones, en muchos casos colectivas, que permiten el avance conjunto (Goh, 2013). Además, y atendiendo al plano metodológico, se vincula a la posibilidad de hibridación de diferentes métodos pedagógicos, lo que abre un gran abanico de posibilidades al docente a la hora de escoger aquellas opciones que mejor considere en cada contexto. A modo de ejemplo, es perfectamente compatible con el aprendizaje cooperativo, que por esencia busca la mejora del grupo a través del aporte de cada una de las individualidades que lo componen (Kimmelman y Lang, 2018). Si el resultado del trabajo va a ser de mayor calidad cuando se delimitan responsabilidades tanto individuales como grupales, parece lógico establecer los mecanismos de consenso que ayuden en la toma de decisiones y ajusten percepciones sobre el nivel de trabajo de cada integrante. En un grupo no se trata de juntar las partes para obtener un mejor resultado, sino de articular procesos, que basados en la autonomía y responsabilidad del estudiante, promuevan retroalimentaciones efectivas que destaquen las posibilidades y detecten las limitaciones. Algo similar sucede con el flipped classroom, que busca la realización del trabajo previo del estudiante para conexionar y reforzar las temáticas abordadas posteriormente en el aula. De nuevo los canales de retroalimentación cobran especial relevancia, no solo en lo que a los contenidos instrumentales se refiere, sino a los procesos de autorregulación, búsqueda y contraste de información con los que se asocia. Esto también se observa con otras metodologías como el aprendizaje basado en proyectos o el aprendizaje servicio, donde además de buscar vínculos que aporten a la sociedad a través de la creación del vínculo de comunidad, se necesitan feedbacks desde diferentes esferas que reconduzcan los fines pretendidos en las actividades (Hortigüela, Abella, Delgado y Ausín, 2018). Es por tanto que es en la hibridación donde se encuentran las mayores posibilidades de aplicar los sistemas de EFyC, combinándolas con metodologías de carácter abierto y participativo. Por lo tanto, y si entendemos la evaluación como la esencia de los procesos educativos, construiremos una forma de entender este elemento curricular, y, por ende, definiremos nuestra identidad profesional como docentes. 


\section{Reflexiones prácticas}

Hemos comprobado cómo, aunque la literatura científica en los últimos años ha avanzado sustancialmente en el ámbito de la evaluación, todavía son muchas las confusiones e incertidumbres que surgen en torno a la implantación de la misma. Por ello, necesitamos seguir trabajando, y lo que es más importante, asumir ciertos acuerdos que nos permitan unificar modos de aplicar esta evaluación en el aula. Estos cambios evaluativos no afectan únicamente a cómo poder calificar de un modo transparente y democrático, ya que se tratan de aspectos pertenecientes a un cambio de paradigma educativo sobre el que hay que seguir trabajando (Hortigüela, Pérez-Pueyo y López-Pastor, 2015). La frase "Dime cómo evalúas y te diré que enseñas" parece que cobra más sentido que nunca hoy en día, donde son diversidad de metodologías las que nos abordan, en muchos casos de manera irreflexiva y excesivamente debido a razones mediáticas. Es por ello que la innovación educativa ha de nacer primeramente en nosotros como docentes, buscando nuestras fortalezas y reconociendo nuestras debilidades con el fin de mejorarlas y adaptarlas de un modo positivo en nuestro contexto. No sirve de nada pensar que nuestras metodologías van a seguir funcionando por el hecho de que lo hicieron siempre, ya que han de estar en continuo avance y adaptándose tanto a las necesidades de nuestros estudiantes como al tipo de sociedad tan cambiante en la que viven (Navas, Salarirche y Sola, 2017). Es preciso ser consciente de que uno no incorpora a sus características personales la crítica, la creatividad o la reflexión de una forma repentina, sino que estas competencias deben de ser trabajadas e interiorizadas a través de diversidad de tareas que promuevan experiencias positivas de aprendizaje (Mazzitelli, Guirado y Laudadio, 2018).

Una de las características más destacables de la $\mathrm{EFyC}$ es su transversalidad, por lo que permite su aplicación y desarrollo en cualquier etapa educativa. Esto permite que, con una clara visión pedagógica transformadora, la evaluación pueda ser cada vez más un verdadero agente de cambio en las aulas. Cuando el docente es el único agente que controla la evaluación y la calificación, hay una gran cantidad de aspectos que nos estamos dejando por el camino. Si compartimos aula con los estudiantes es vital construir el aprendizaje de una forma retroalimentada, debiendo ser el alumno pleno conocedor de las "reglas del juego" desde el inicio (Hortigüela y Pérez-Pueyo, 2016). Podríamos pensar entonces cuáles son los principales motivos por los que el profesorado reconoce no adentrarse en estos sistemas de evaluación: a) les es más sencillo y menos traumático responsabilizarse exclusivamente de la evaluación; b) les supone una menor carga de trabajo al utilizar la calificación como principal vía de actuación; c) sienten que tienen un mayor control del aula al ser ellos los que tomen todas las decisiones evaluativas (López-Lozano, Solís y Azcárate, 2018). Si atendemos a estos aspectos, observamos claramente cómo están fundamentados en una lógica de tradición y cultura, donde el profesor es el único que sabe y decide exclusivamente sobre la nota que tiene el estudiante. Este tipo de funcionamiento también suele estar amparado por las familias, siendo además socialmente aceptado. Parece que lo verdaderamente importante es el número (nota) que se obtiene al finalizar un trimestre/semestre, sin cuestionarnos los factores más relevantes como el nivel de aprendizaje adquirido, su percepción de utilidad y transferencia o las razones de una mayor o menor implicación y consciencia del proceso de enseñanza. La mejor manera para romper ese paradigma tradicional es entender y concienciar al docente, de que fórmulas participativas, en este caso a través de la evaluación, son necesarias para hacer de la educación algo compartido (Springett, 2017) 
Si bien la EFyC puede ser aplicada dentro de cualquier etapa, lo mismo sucede al poderla abordar en cualquier área y especialidad. De hecho, un aspecto de verdadero interés radica en poder investigar sobre sus propios efectos. Esto, que podría parecer que tuviera más cabida en el ámbito universitario, se puede desarrollar sin problemas en las etapas de primaria y secundaria. Investigar sobre la propia práctica docente y sobre los procesos de enseñanza a través de ciclos de investigación-acción es una de las mejores maneras de ser consciente de los resultados de la enseñanza. Lo importante es saber lo que se quiere obtener y la manera de reconfigurar el día a día en las aulas. Esa evolución de la práctica profesional únicamente se obtiene si registramos cada uno de nuestros avances, los de los alumnos y elaboramos estrategias acordes que favorezcan la conexión con sus intereses (Barnes y Brownell, 2016).

A lo largo de su vida, el individuo va aprendiendo a través de diferentes canales y ámbitos educativos, tanto el formal como el informal. En el informal, más decisivo que el formal en muchos casos, uno decide cómo, cuándo y de qué manera interacciona con los elementos que le rodean. Esto se traduce en que en la vida uno aprende a través de lo que le motiva. En función de esto, podríamos preguntarnos por qué el aprendizaje formal de la escuela no se fundamenta y se reconceptualiza bajo ejes claros motivantes que vayan más allá de los propios contenidos curriculares (Subasi y Tas, 2016). Suele otorgarse el peso principal de la "buena educación" a la cantidad de contenidos que un alumno puede asumir, en muchos casos ingerir, cuando la realidad nos demuestra día tras día que esto no se traduce en la adquisición de verdaderas competencias. La clave de nuevo se centra más en el cómo que en el qué, siendo necesario el desarrollo de la totalidad de capacidades del estudiante a través de la propuesta de proyectos de trabajo que conexionen con la realidad (Sampaio y Leite, 2017). Esto no está reñido con que se deje de lado el esfuerzo, la dedicación o la constancia, pero es que difícilmente alguien puede dedicar tiempo a algo que le desmotiva totalmente desde el inicio. La creación de ambientes de trabajo positivos, sostenidos bajo buenos climas de grupo, pueden suponer el cambio hacia el aprendizaje motivador (Bakhtiar, Webster y Hadwin, 2018).

Por todo ello, la evaluación, entendida como uno de los ejes fundamentales que articula el proceso educativo, ha de entenderse como un agente socializador que permita la plena consciencia sobre el aprendizaje (Hortigüela, Pérez-Pueyo y Abella, 2015c). Esta consciencia solamente alcanza su máxima plenitud si el alumnado participa intencionadamente en la misma, no solo en su inicio y final, sino a lo largo de todo el proceso de enseñanza. Como ya se destacó con anterioridad, hay que tener presente que la evaluación siempre tiene que ser un aporte, y no puede convertirse en una traba. Por ello, y debido a que los procesos de EFyC suponen una mayor carga de trabajo para el profesorado en relación a las vías tradicionales, hay que atender al criterio de la viabilidad. Un proceso didáctico que no es sostenible en el tiempo no garantiza ninguna efectividad ni credibilidad. Por ello, y para los profesores que se inicien en estos sistemas, tienen que hacer un ejercicio de adecuación y proporcionalidad en función de su realidad: número de alumnos por aula, duración de la asignatura, tipo y número de tareas demandadas...En esto, y como en cualquier otra metodología didáctica, hay que evadirse de radicalismos, no habiendo nada estrictamente bueno o malo. Lo mejor es aquello que tiene efectos positivos de aprendizaje y de transformación social en el aquel contexto en el que se lleva a cabo (Craft y Chappell, 2016). Por tanto, técnicas evaluativas que funcionan con 
determinados grupos o en determinados centros educativos han de ser adaptadas en cursos posteriores.

Lo más importante, al diseñar la evaluación de cualquier proceso educativo, es que garantice las condiciones, en el marco temporal en el que se lleva a cabo, de un aprendizaje verdaderamente competencial y consciente. Un aprendizaje que, a través del fomento de la autonomía y la de capacidad organizativa del estudiante, le permita concebir su verdadero aporte a la solución de tareas mediante el disfrute. Sí, se puede aprender disfrutando. De hecho, estudios como el de Siry and Brendel (2016) demuestran que el trabajo de las emociones es directamente proporcional al vínculo afectivo que se genera entre docente y estudiante, y esto repercute en la implicación hacia los contenidos impartidos. Basándonos en esto, los feedbacks aplicados en la evaluación han de tener un componente altamente constructivo y basarse fundamentalmente en las posibilidades de mejora. Si esto es así, y se implanta con asiduidad y normalidad, pasaremos del miedo a intervenir por el fallo o risa de los demás a la participación para la mejora común, esencia esta del aprendizaje (Mandouit, 2018).

\section{Conclusiones}

En el presente manuscrito hemos establecido una reflexión argumentada acerca de los principales errores que suelen cometerse a la hora de implantar los sistemas de EFyC. Fundamentalmente, estos se centran en la confusión de la evaluación con la calificación, la falta de transparencia y delimitación de los criterios de antemano, la no implicación del alumnado en los procesos evaluativos y la no utilización de la evaluación como una herramienta puramente pedagógica. Esto supone un aporte significativo a la literatura, ya que, si bien esta aborda los beneficios y problemáticas de esta evaluación, no se han analizado desde este enfoque. Por lo tanto, parece oportuno que un manuscrito de este tipo tenga cabida en la actualidad. Si bien estamos abordando un artículo de carácter descriptivo y reflexivo, a partir del mismo se abren diversas líneas de investigación futuras. Una de ellas sería investigar específicamente en cada uno de los 7 ámbitos abordados, pudiendo establecer así comparaciones a partir de experiencias concretas. También pudieran realizarse meta-análisis sobre algunos de los tópicos tratados, con el fin de profundizar en la literatura existente hasta la actualidad. Consideramos que este artículo podría ser de interés para todos aquellos docentes, de cualquier etapa educativa, interesados en el ámbito de la evaluación y en la educación democrática. También para todos aquellos políticos y legisladores en el ámbito educativo, valorando la importancia que tienen los métodos de enseñanza dentro de los currículos actuales. En este sentido, y si verdaderamente otorgamos la relevancia real que tiene la educación, no podemos seguir evaluando de la misma manera.

\section{Referencias}

Alcaraz, N., Navas, M. y Solac, M. (2012). La voz del alumnado en los procesos de evaluación docente universitaria. Revista Iberoamericana de Evaluación Educativa, 5(2), 26-39.

Alonzo, A. (2018). An Argument for Formative Assessment with Science Learning Progressions. Applied Measurement in Education, 31(2), 104-112.

https://doi.org/10.1080/08957347.2017.1408630 
Ashgar, A. (2010). Reciprocal Peer Coaching and Its Use as a Formative Assessment Strategy for First-Year Students. Assessment \& Evaluation in Higher Education, 35(4), 403-41 7.

Bakhtiar, A., Webster, E. A. y Hadwin, A. F. (2018). Regulation and Socio-Emotional Interactions in a Positive and a Negative Group Climate. Metacognition and Learning, 13(1), 57-90. https://doi.org/10.1007/s1 1409-017-9178-x

Barnes, M. E. y Brownell, S. E. (2016). Practices and Perspectives of College Instructors on Addressing Religious Beliefs When Teaching Evolution. CBE - Life Sciences Education, 15(1), 54-66.

Grosas, A., Raju, S., Schuett, B., Chuck, J. y Millar, T. (2016). Determining if active learning through a formative assessment process translates to better performance in summative assessment. Studies in Higher Education, 41(9), 1595-1611. https://doi.org/10.1080/03075079.2014.988704

Calkins, A. et al. (2018). Five Elements for Assessment Design and Use to Support Student Autonomy. Students at the Center: Deeper Learning Research Serie. Jobs for the Future, 25(1), 12-14.

Cano, E. (2008). La evaluación por competencias en la educación superior. Profesorado. Revista de Currículum y Formación de Profesorado, 12(3), 1-16.

Chiappe, A., Pinto, R. y Arias, V. (2016). Open Assessment of Learning: A Meta-Synthesis. International Review of Research in Open and Distributed Learning, 17(6), 44-61.

Craft, A. R. y Chappell, K. A. (2016). Possibility Thinking and Social Change in Primary Schools. Education, 44(4), 407-425. https://doi.org/10.1080/03004279.2014.961947

Delgado, V., Hortigüela, D., Ausín, V. y Abella, V. (2018). El Blog como Instrumento de Mejora para la Autorregulación del Aprendizaje del Estudiante Universitario. Estudios Pedagógicos, 44(2), 171-184.

Fraile, A., López-Pastor, V., Castejón, J. y Romero, R. (2013). La evaluación formativa en docencia universitaria y el rendimiento académico del alumnado. Aula Abierta, 41(2), 23-34.

Goh, A. (2013). The Significance of Social Relationships in Learning to Become a Vocational and Technical Education Teacher: A Case Study of Three Individuals. Studies in Continuing Education, 35(3), 366-378. https://doi.org/10.1080/0158037X.2013.770390

Gutiérrez, C., Hortigüela, D., Peral, Z. y Pérez-Pueyo, A. (2018). Percepciones de Alumnos del Grado en Maestro en Educación Primaria con Mención en Educación Física sobre la Adquisición de Competencias. Estudios Pedagógicos, 44(2), 223-239.

Hortigüela, D. y Pérez-Pueyo, A. (2016). La evaluación entre iguales como herramienta para la mejora de la práctica docente. Revista Opción, 32(7), 865-879.

Hortigüela, D., Abella, V., Delgado, V. y Ausín, V. (2018). Valoración del aprendizaje obtenido en la formación inicial del profesorado en función del enfoque metodológico. Profesorado. Revista de Currículo y Formación del Profesorado, 22(2), 183-202.

Hortigüela, D., Palacios, A. y López-Pastor, V. M. (2018). The impact of formative and shared or coassessment on the acquisition of transversal competences in higher education. Assessment E Evaluation in Higher Education, 43(3), 1-14. https://doi.org/10.1080/02602938.2018.1530341

Hortigüela, D., Pérez-Pueyo, A. y Abella, V. (2015a). ¿De qué manera se implica el alumnado en el aprendizaje? Análisis de su percepción en procesos de evaluación formativa. Revista de Investigación en Educación, 13(1), 88-104. 
Hortigüela, D., Pérez Pueyo, A. y Abella, V. (2015b). Perspectiva del alumnado sobre la evaluación tradicional y la evaluación formativa. Contraste de grupos en las mismas asignaturas. REICE. Revista Iberoamericana sobre Calidad, Eficacia y Cambio en Educación, 13(1), 35-48.

Hortigüela, D., Pérez-Pueyo, A. y Abella, V. (2015c). ¿Cómo influye el sistema de evaluación en la percepción del alumnado? @TIC revista d' innovació educativa, 14, 82-89. https://doi.org/10.7203/attic.14.4170.

Hortigüela, D., Pérez-Pueyo, A. y López-Pastor, V. M. (2015). Implicación y regulación del trabajo del alumnado en los sistemas de evaluación formativa en educación superior. RELIEVE, 21(1), 1-5. https://doi.org/10.7203/relieve.21.1.5171

Hortigüela, D., Fernández-Río, J., Castejón, J. y Pérez-Pueyo, A. (2017). Formative assessment, work regulation, organization, engagement, tracking and attendance in Spanish Universities. Revista Electrónica Interuniversitaria de Formación del Profesorado, 20(3), 49-63. https://doi.org/10.6018/reifop.20.3.268681

Joughin, G., Dawson, P. y Boud, D. (2017). Improving Assessment Tasks through Addressing Our Unconscious Limits to Change. Assessment E Evaluation in Higher Education, 42(8), 12211232. https://doi.org/10.1080/02602938.2016.1257689

Keely, P. (2016). Formative Assessment Probes: Big and Small Seeds. Linking Formative Assessment Probes to the Scientific Practices. Science and Children, 53(9), 20-21.

Kimmelman, N. y Lang, J. (2018). Linkage within teacher education: cooperative learning of teachers and student teachers. European Journal of Teacher Education, 42(1), 52-64. https://doi.org/10.1080/02619768.2018.1547376

Klenowski, V., Askew, S. y Carnell, E. (2006). Portfolios for learning, assessment and professional development in higher education. Journal Assessment \& Evaluation in Higher Education, 31(3), 267-286. https://doi.org/10.1080/02602930500352816

López-Lozano, L., Solís, E. y Azcárate, P. (2018). Evolution of Ideas about Assessment in Science: Incidence of a Formative Process. Research in Science Education, 48(5), 915-937. https://doi.org/10.1007/s11165-016-9591-1

López-Pastor, V. M. (2008). Desarrollando sistemas de evaluación formativa y compartida en la docencia universitaria. Análisis de resultados de su puesta en práctica en la formación inicial del profesorado. European Journal of Teacher Education, 31(3), 293-311. https://doi.org/10.1080/02619760802208452

López-Pastor, V. M. y Sicilia-Camacho, A. (2017). Formative and shared assessment in higher education. Lessons learned and challenges for the future. Assessment E Evaluation in Higher Education, 42(1), 77-97. https://doi.org/10.1080/02602938.2015.1083535

Lorente-Catalán, E. y Kirk, D. (2014). Making the Case for Democratic Assessment Practices within a Critical Pedagogy of Physical Education Teacher Education. European Physical Education Review, 20(1), 104-119. https://doi.org/10.1177/1356336X13496004

Mandouit, L. (2018). Using Student Feedback to Improve Teaching. Educational Action Research, 26(5), 755-769. https://doi.org/10.1080/09650792.2018.1426470

Mazzitelli, C. A. Guirado, A. y Laudadio, M. J. (2018). Estilos de Enseñanza y Representaciones sobre Evaluación y Aprendizaje. Revista Iberoamericana de Evaluación Educativa, 11(1), 5772. https://doi.org/10.15366/riee2018.11.1.004

Meusen-Beekman, K. D., Joosten-ten Brinke, D. y Boshuizen, H. (2015). Developing Young Adolescents' Self-Regulation by Means of Formative Assessment: A Theoretical 
Perspective. Cogent Education, 2(1), 107-123.

https://doi.org/10.1080/2331186X.2015.1071233

Navas, M., Salarirche, N. y Sola, M. (2017). Evaluación y Pruebas Estandarizadas: Una Reflexión sobre el Sentido, Utilidad y Efectos de estas Pruebas en el Campo Educativo. Revista Iberoamericana de Evaluación Educativa, 10(1), 51-67. https://doi.org/10.15366/riee2017.10.1.003

Purcell, B. M. (2014). Use of Formative Classroom Assessment Techniques in a Project Management Course. Journal of Case Studies in Accreditation and Assessment, 12(1), 13-22.

Sampaio, M. y Leite, C. (2017). From Curricular Justice to Educational Improvement: What Is the Role of Schools' Self-Evaluation? Improving Schools, 20(1), 62-75. https://doi.org/10.1177/1365480216688553

Serrano, R. (2010). Pensamientos del profesor: un acercamiento a las creencias y concepciones sobre el proceso de enseñanza-aprendizaje en la Educación Superior. Revista de Educación, $352,267-287$.

Shepard, L. A., Penuel, W. R. y Pellegrino, J. W. (2018). Using Learning and Motivation Theories to Coherently Link Formative Assessment, Grading Practices, and Large-Scale Assessment. Educational Measurement: Issues and Practice, 37(1), 21-34.

Siry, C. y Brendel, M. (2016). The Inseparable Role of Emotions in the Teaching and Learning of Primary School Science. Cultural Studies of Science Education, 11(3), 803-815. https://doi.org/10.1007/s1 1422-016-9781-1

Springett, J. (2017). Impact in Participatory Health Research: What Can We Learn from Research on Participatory Evaluation? Educational Action Research, 25(4), 560-574. https://doi.org/10.1080/09650792.2017.1342554

Subasi, M. y Tas, Y. (2016). The Role of Motivating Tasks and Personal Goal Orientations in Students' Coping Strategies in Science. Asia-Pacific Forum on Science Learning and Teaching, $17(2), 45-57$.

Wang, W. (2017). Using Rubrics in Student Self-Assessment: Student Perceptions in the English as a Foreign Language Writing Context. Assessment $\underbrace{2}$ Evaluation in Higher Education, 42(8), 1280-1292. https://doi.org/10.1080/02602938.2016.1261993

Wanner, T. y Palmer, E. (2018). Formative Self- and Peer Assessment for Improved Student Learning: The Crucial Factors of Design, Teacher Participation and Feedback. Assessment E Evaluation in Higher Education, 43(7), 1032-1047. https://doi.org/10.1080/02602938.2018.1427698

Wei, W. (2014). Using summative and formative assessments to evaluate EFL teachers' teaching performance. Assessment $E^{2}$ Evaluation in Higher Education, 4O(4), 611-623. https://doi.org/ 10.1080/02602938.2014.939609

Wilkie, K. J. (2016). Using Challenging Tasks for Formative Assessment on Quadratic Functions with Senior Secondary Students. Australian Mathematics Teacher, 72(1), 30-40. 


\section{Cv de los autores}

\section{David Hortigüela Alcalá}

Doctor en Educación por la Universidad de Burgos. Es Director del Área de Didáctica de la Expresión Corporal de la Universidad de Burgos y Secretario del Departamento de Didácticas Específicas. Sus líneas de investigación se centran en la evaluación formativa y compartida, los modelos pedagógicos de enseñanza de la educación física, las metodologias activas y participativas y el aprendizaje competencial. Sus investigaciones pueden encontrarse en: http://bit.ly/dhortigüelacv. ORCID ID: 0000-0001-5951-758X. Email: dhortiguela@ubu.es

\section{Ángel Pérez Pueyo}

Doctor en Educación por la Universidad de León. Es Director del Área de Extensión Universitaria de la Universidad de León. Sus líneas de investigación se centran en la evaluación formativa y compartida, la didáctica de la educación física, el trabajo de las competencias clave y la autorregulación del aprendizaje. Sus investigaciones pueden encontrarse en: http://bit.ly/pueyo_cv. ORCID ID: O000-0002-3177-2199. Email: angel.perez.pueyo@unileon.es

\section{Gustavo González Calvo}

Doctor en Ciencias de la Educación por la Universidad de Valladolid. Es profesor del Departamento de Didáctica de la Expresión Corporal en la Facultad de Educación de Palencia (Universidad de Valladolid). Sus líneas de investigación se centran en el desarrollo de la identidad personal y profesional del profesorado de Educación Física, las etnografías escolares, el estudio de las subjetividades corporales y la investigación a través de historias de vida. Sus investigaciones pueden encontrarse en: http://bit.ly/Gustavo_gonz. ORCID ID: Oo00-0002-4637-0168. Email: gustavo.gonzalez@uva.es 